\title{
Study on Frequency Characteristics of Rotor Systems for Fault Detection Using Variational Mode Decomposition
}

\author{
Kai Chen, ${ }^{1,2}$ Xin-Cong Zhou, ${ }^{1,2}$ Jun-Qiang Fang, ${ }^{1,2}$ and Li Qin ${ }^{3}$ \\ ${ }^{1}$ Key Laboratory of High Performance Ship Technology (Wuhan University of Technology), Ministry of Education, \\ Wuhan 430063, China \\ ${ }^{2}$ Reliability Engineering Institute, School of Energy and Power Engineering, Wuhan University of Technology, \\ Wuhan 430063, China \\ ${ }^{3}$ School of Machinery and Automation, Wuhan University of Science and Technology, Wuhan 430081, China \\ Correspondence should be addressed to Kai Chen; kaichen@whut.edu.cn
}

Received 28 April 2017; Revised 10 July 2017; Accepted 16 July 2017; Published 14 August 2017

Academic Editor: Adam Glowacz

Copyright (C) 2017 Kai Chen et al. This is an open access article distributed under the Creative Commons Attribution License, which permits unrestricted use, distribution, and reproduction in any medium, provided the original work is properly cited.

\begin{abstract}
Due to the complicated structure, vibration signal of rotating machinery is multicomponent with nonstationary and nonlinear features, so it is difficult to diagnose faults effectively. Therefore, effective extraction of vibration signal characteristics is the key to diagnose the faults of rotating machinery. Mode mixing and illusive components existed in some conventional methods, such as EMD and EEMD, which leads to misdiagnosis in extracting signals. Given these reasons, a new fault diagnosis method, namely, variation mode decomposition (VMD), was proposed in this paper. VMD is a newly developed technique for adaptive signal decomposition, which can decompose a multicomponent signal into a series of quasi-orthogonal intrinsic mode functions (IMFs) simultaneously, corresponding to the components of signal clearly. To further research on VMD method, the advantages and characteristics of VMD are investigated via numerical simulations. VMD is then applied to detect oil whirl and oil whip for rotor systems fault diagnosis via practical vibration signal. The experimental results demonstrate the effectiveness of VMD method.
\end{abstract}

\section{Introduction}

For a long time, faults of rotating machinery were mainly diagnosed by spectrum analysis method of vibration signal, which determine the failure by analyzing the frequency spectrum or frequency characteristics of vibration signals [1]. Practically, the vibration signal is generally nonstationary and nonperiodic when the fault occurs, so the traditional FFT cannot meet the needs of the time-varying and nonstationary signals detection and time-frequency analysis [2].

Like many fault signals, the vibration signal of rotor system is a typical nonlinear and nonstationary time-varying signal whose frequency components change over time [3]. The feature extracting of fault signal not only affects the accuracy of fault diagnosis, but also may lead to misdiagnosis. Therefore, effective extraction of vibration signal characteristics is the key to diagnose the faults of rotating machinery [4].
Analysis, processing, and feature extraction of nonlinear and nonstationary signal always are one of the hot topics concerning engineers and researchers [5-7]. EMD is an effective signal analysis method which is suitable for dealing with nonlinear and nonstationary signals [8]. It consists in a local and fully data-driven separation of a signal with fast and slow oscillations. However, EMD experiences some problems, such as end effect and mode mixing [9]. To overcome shortcomings, the ensemble empirical mode decomposition (EEMD) was proposed [10], which can suppress the appearance of modal mixing. But both of them lack theoretical support. Besides being recursive decomposition, error of envelope line will be spread; thus they cannot eliminate the problem of modal mixing completely.

Dragomiretskiy and Zosso [11] have proposed a new adaptive decomposition method called variational mode decomposition (VMD) in 2014, which can nonrecursively 
decompose a signal into a number of intrinsic mode functions (IMFs). In the process of decomposition, by using alternate direction multipliers method (ADMM), the center frequency and bandwidth of each modal were updated, to search the optimal solution of the signal decomposition. The updating process of each mode is carried out synchronously and makes the component of each modal in the corresponding baseband, finally, to achieve the effective decomposition of the signal. Compared with EMD and EEMD, VMD does not strip the signal step by step, but decomposes the signal synchronously. It is nonrecursive and reduces the spread of error. This method also has a good theoretical basis which is easy to understand. Besides, it has stronger robustness to noise [12, 13]. With its advantages, VMD is a wide application in the research field of signal denoising [14, 15], feature extraction [16, 17], image processing [18], energy and economics price forecasting $[19,20]$, speech signals detection [21], and especially in fault diagnosis [22-25]. However, these literatures mainly focus on the advantages of VMD comparing with the EMD and EEMD, rather than investigating the own capabilities of the VMD in signal analysis. The research of VMD needs to be conducted in depth. For this reason, a further investigation of the VMD is conducted in this paper via both numerical simulation and experimental approaches.

In this paper, the characteristics of VMD are investigated based on the theoretical of VMD, as well as the advantages of VMD. And then, VMD is applied to detect the fault of oil whirl and oil whip in rotor systems. This provides an effective solution for fault diagnosis of rotor system.

\section{Variational Mode Decomposition}

2.1. EMD and EEMD Method. EMD is a time-frequency signal analysis method for nonlinear signals, which can decompose the data adaptively and obtain a series of IMFs [9]. These IMFs reflect the characteristics of the signal itself. EMD algorithm is very suitable for analyzing the nonstationary signal.

Each signal could be decomposed into a number of intrinsic mode functions (IMFs), each of which must satisfy the following definition [9]:

(1) In the whole data set, the number of extrema and the number of zero-crossings must either be equal or differ at most by one.

(2) At any point, the mean value of the envelope defined by local maxima and the envelope defined by the local minima is zero.

With the definition, any signal $x(t)$ can be decomposed as follows [9]:

(1) Identify all local maxima and all local minima in the signal $x(t)$.

(2) Connect all local maxima and all local minima by using a cubic spline line as the upper envelope $e_{u}(t)$ and the lower envelope $e_{l}(t)$, respectively. The mean $m_{1}(t)$ of upper and low envelope is calculated by using the equation

$$
m_{1}(t)=\frac{\left(e_{u}(t)+e_{l}(t)\right)}{2} .
$$

(3) Calculate the equation

$$
h_{1}(t)=x(t)-m_{1}(t)
$$

and examine whether the resultant $h_{1}(t)$ is an IMF satisfying the two aforementioned conditions. If $h_{1}(t)$ is not an IMF, regard $h_{1}(t)$ as original signal and repeat steps (1)-(3) until $h_{1}(t)$ is an IMF.

(4) Separate the first IMF from $x(t)$ and then get the residual component $r_{1}(t)$ :

$$
r_{1}(t)=x(t)-h_{1}(t) .
$$

Treat $r_{1}(t)$ as original data and repeat the above processes; therefore the second IMF $h_{2}(t)$ is obtained. Iterate the previous calculations $n$ times and $n$ IMFs of the signal could be got. Then

$$
r_{n}(t)=r_{n-1}(t)-h_{n}(t) .
$$

The decomposition process can be stopped when the final residual component $r_{1}(t)$ is a monotonic function or a constant from which no more IMF can be extracted.

(5) The original signal $x(t)$ finally can be expressed as

$$
x(t)=\sum_{i=1}^{n} h_{i}(t)+r_{n}(t) .
$$

Thus, one can achieve a decomposition of the signal into $n$-empirical modes, and a residue $r_{n}(t)$, which is the mean trend of $x(t)$. The IMFs $h_{1}(t), h_{2}(t), \ldots, h_{n}(t)$ include different frequency bands ranging from high to low.

EMD is a self-adaptive signal decomposition method, and the obtained IMFs have the advantages of mutual orthogonality. However, practice has disclosed that the EMD also shows the following defects in signal processing and feature extraction.

(1) No Mathematical Foundation. Without strict mathematical proof and mathematical model, EMD lacks theoretical support. And there is no valid way to stop the decomposition process in the sifting process of EMD.

(2) End Effect. In the decomposition process, endpoint will be used as an extreme point for fitting the upper and lower envelopes. But if the endpoint is not an extreme point, the fitted curve using cubic spline line will have some error. This error will impact on each subsequent decomposition step; the error will accumulate gradually and then is likely to pollute the internal decomposed results from both ends.

(3) Mode Mixing. Mode mixing occurs in EMD; when steps change, pulse interference and outside noise will show in the signal. There are two forms of mode mixing; one form is a single IMF including oscillations of dramatically disparate scales and the other is a component of a similar scale residing in different IMFs. Once the mode mixing is generated, subsequent decomposition step will be influenced, even making IMFs meaningless in physical interpretation.

To alleviate the mode mixing problem occurring in EMD, an ensemble empirical mode decomposition (EEMD) 
is presented [26]. The essential essence of EEMD algorithm is decomposing the original signal added Gaussian noise repeatedly by EMD method, and the original vibration data is decomposed into a series of IMFs with different scales and continuous characteristics because of the characteristic of frequency uniform distribution of Gaussian white noise, which can suppress the appearance of modal mixing.

2.2. VMD Method. VMD is a new signal analysis method for nonlinear and nonstationary signal, which aims to decompose the signal into different discrete modes [11]. The VMD has a solid mathematical foundation, which is able to decompose a signal into an ensemble of band-limited intrinsic mode functions (IMFs) simultaneously. IMFs are redefined as amplitude modulated frequency modulated (AM-FM) signals, written as

$$
u_{k}(t)=A_{k}(t) \cos \left(\varphi_{k}(t)\right),
$$

where $u_{k}(t)$ is IMF, $A_{k}(t)$ is nonnegative envelope, and $\varphi_{k}(t)$ is the phase and a nondecreasing function.

The core of VMD is to construct and solve the variational problem, the decomposition process of $\mathrm{VMD}$ algorithm is the solution of the variational problem. There are three important concepts in the signal processing, Wiener Filtering, Hilbert transform, and frequency mixing, which constitute the building blocks of VMD model.

Assume each mode $u_{k}$ is band-limited and compactly distributed around with a center frequency $w_{k}$, so the solution of variational problem turns to seeking for $k$ modes $u_{k}$ to make the sum of the bandwidth minimized, while the constraint condition is the sum of modes equal to the input signal $f$. The principle of the VMD algorithm is the following:

(1) The analytic signal of mode $u_{k}$ was computed by means of Hilbert transform, so obtain the unilateral spectrum of the analytic signal.

$$
\delta(t)+\frac{j}{\pi(t)} * u_{k}(t)
$$

(2) Shift the frequency spectrum of each mode to the respective estimated central frequency.

$$
\left[\delta(t)+\frac{j}{\pi(t)} * u_{k}(t)\right] \cdot e^{-j w_{k} t} .
$$

(3) The bandwidth is estimated through the Gaussian smoothness of the demodulated signal, that is, the squared $L^{2}$-norm of the gradient. The resulting constrained variational problem is given by the following:

$$
\begin{aligned}
\min _{\left\{u_{k}, w_{k}\right\}} & \left\{\sum_{k}\left\|\partial_{t}\left[\delta(t)+\frac{j}{\pi(t)} * u_{k}(t)\right] \cdot e^{-j w_{k} t}\right\|_{2}^{2}\right\} \\
\text { s.t } & \sum_{k} u_{k}=f,
\end{aligned}
$$

where $\left\{u_{k}\right\}:=\left\{u_{1}, \ldots, u_{k}\right\}$ and $\left\{w_{k}\right\}:=\left\{w_{1}, \ldots, w_{k}\right\}$ are the identified set of modes and their central frequencies.
(4) A quadratic penalty term $\alpha$ and a Lagrangian multiplier factor $\lambda(t)$ are introduced in order to render the problem unconstrained. The quadratic penalty is a classic way to encourage reconstruction fidelity, and the Lagrangian multipliers are a common way of enforcing constraints strictly. The augmented Lagrangian is shown as follows:

$$
\begin{aligned}
& L\left(\left\{u_{k}\right\},\left\{w_{k}\right\}, \lambda\right) \\
& =\alpha \sum_{k}\left\|\left[\delta(t)+\frac{j}{\pi(t)} * u_{k}(t)\right] \cdot e^{-j w_{k} t}\right\|_{2}^{2} \\
& +\left\|f(t)-\sum_{k} u_{k}(t)\right\|_{2}^{2} \\
& +\left\langle\lambda(t), f(t)-\sum_{k} u_{k}(t)\right\rangle .
\end{aligned}
$$

(5) An alternate direction multipliers method (ADMM) is applied to solve the original minimization problem, to find the saddle point of the augmented Lagrange expression via updating $u_{k}^{n+1}, w_{k}^{n+1}$, and $\lambda_{k}^{n+1}$ alternately. So the signal was decomposed into different discrete modes adaptively.

The flowchart of VMD methods is shown in Figure 1, and the detailed steps are as follows.

(1) Initialize $\left\{\hat{u}_{k}^{1}\right\},\left\{w_{k}^{1}\right\}, \hat{\lambda}^{1}$ and $n=0$.

(2) $n=n+1$; update $\widehat{u}_{k}$ and $w_{k}$ according to (11) and (12).

$$
\begin{aligned}
\widehat{u}_{k}^{n+1}(w) & =\frac{\widehat{f}(w)-\sum_{i \neq k} \widehat{u}_{i}^{n}(w)+\widehat{\lambda}^{n}(w) / 2}{1+2 \alpha\left(w-w_{k}^{n}\right)^{2}}, \\
w_{k}^{n+1} & =\frac{\int_{0}^{\infty} w\left|\widehat{u}_{k}^{n+1}(w)\right|^{2} d w}{\int_{0}^{\infty}\left|\widehat{u}_{k}^{n+1}(w)\right|^{2} d w},
\end{aligned}
$$

where $k=\{1,2, \ldots, K\}$.

(3) Update $\lambda$.

$$
\widehat{\lambda}^{n+1}(w)=\widehat{\lambda}^{n}(w)+\tau\left(\widehat{f}(w)-\sum_{k} \widehat{u}_{k}^{n+1}(w)\right) .
$$

(4) Repeat steps (2) and (3), until the following convergence condition is satisfied:

$$
\frac{\sum_{k}\left\|\widehat{u}_{k}^{n+1}-\widehat{u}_{k}^{n}\right\|_{2}^{2}}{\left\|\widehat{u}_{k}^{n}\right\|_{2}^{2}}<\varepsilon,
$$

where $\varepsilon \geq 0$ is the convergence tolerance.

To show the advantage of VMD, the simulated signal in (15) is decomposed by using EMD, EEMD, and VMD, respectively; the results are shown in Figure 2.

$$
\begin{aligned}
& y_{1}=\sin (2 \pi 30 t), \\
& y_{2}=\sin (2 \pi 10 t) *(t>0.2), \\
& y_{3}=\sin (2 \pi 50 t) *(t<0.2), \\
& y=y_{1}+y_{2}+y_{3} .
\end{aligned}
$$




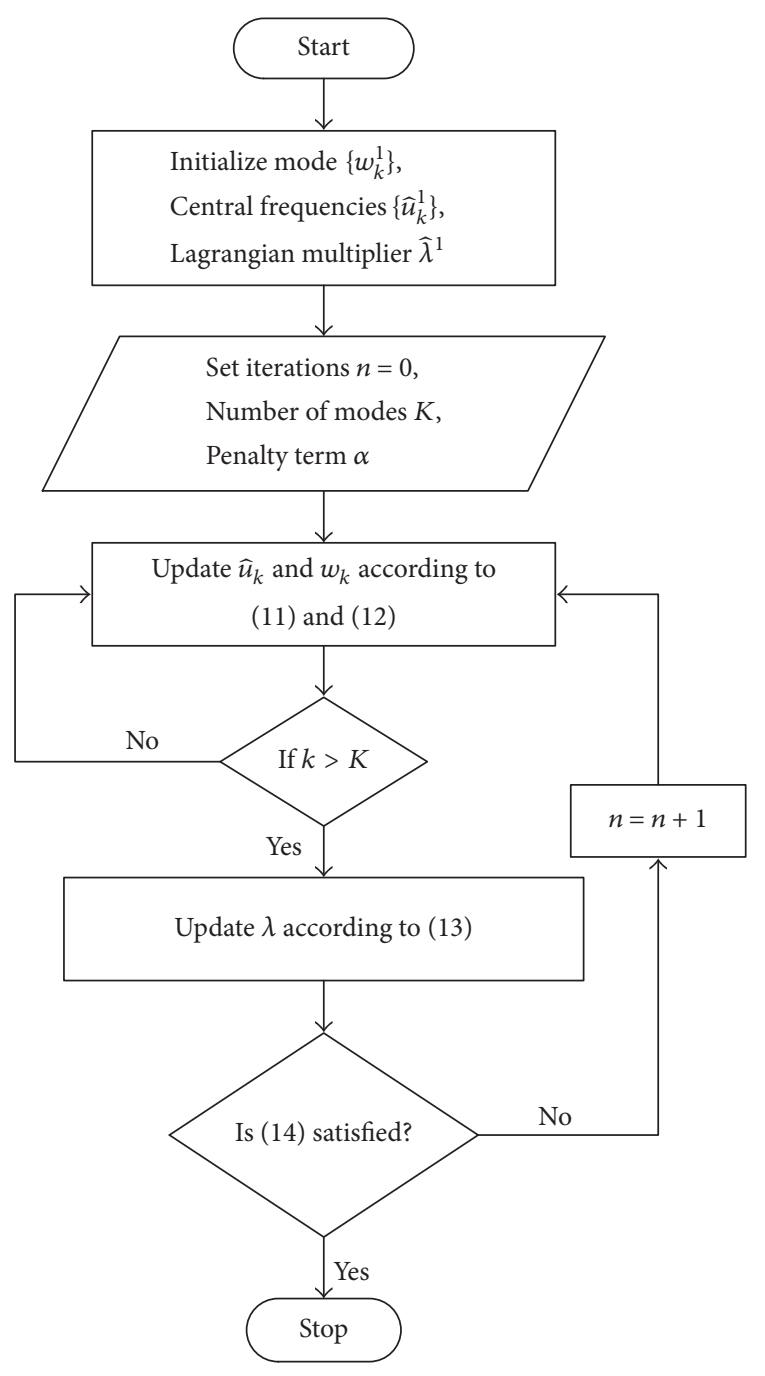

FIGURE 1: Flowchart of VMD.

It can be seen from Figure 2 that the three components of original signal were decomposed by VMD without mode mixing, while it is decomposed by VMD completely. The IMF2, IMF3, and IMF4 decomposed by EEMD are basically the same as the three components in the original signal, but mode mixing was not completely eliminated, and end effect still exists; furthermore, there is a high-frequency component IMF1. And the IMFs decomposed by EMD are hard to distinguish while the mode mixing still exists. In summary, VMD is better than EEMD and EMD in feature extraction of vibration signal.

\section{Characteristics of VMD}

Compared to EMD and EEMD, VMD has advantages in solving the problem of mode mixing and end effect. What is more, according to the principle and decomposition steps of $\mathrm{VMD}$, the following characteristic of VMD can be obtained.

(1) Adaptivity. Unlike wavelet analysis which needs to select the best wavelet base, the signal was decomposed by VMD without prior knowledge. Various signals can be decomposed into different IMFs according to information about the signal itself, and the IMFs correspond to the components of different frequency range and center frequency in the signal. This reflects adaptability of the VMD method.

(2) Reconfigurability. As mentioned before, the core of VMD is to solve the variational constraint problem, while the constraint condition is the sum of modes equal to the input signal. So the original signal can be reconstructed by the IMFs decomposed by the VMD, which was proved by formula (9) theoretically. The simulation signal of (15) was taken as an example to show that VMD is reconfigurable. The original signal, the reconstructed signal, and error curve are shown in Figure 3. It can be seen from the figure that the error is very small to explain the reconfigurability of VMD.

(3) Smoothing Filtering. VMD also has certain filtering characteristics. The simulation signal of (15) added a random noise which was decomposed by the VMD and reconstructed. The time and frequency domain of the original simulation signal, the simulation signal added noise, and the reconstructed signal were illustrated in Figure 4.

By comparing the original signal and the reconstructed signal, the added noise was eliminated, and the characteristic peaks of spectrum were more prominent and obvious after reconstitution. And, in the time domain, fluctuating character of the reconstructed signal is more clear than the signal added noise, which is hard to identify. VMD does have the smoothing filter characteristic.

This can also be deduced from the VMD algorithm in theory. It is observed from formula (11) that the quadratic penalty term $\alpha$ is in the denominator. When $\alpha$ increases, the bandwidth of each mode will be decreased, the spectrum becomes smooth, and the filtered and smoothed modal components are discarded as noise.

(4) Orthogonality. A series of modal components (IMFs) were decomposed by VMD; each modal component represents a feature of the original signal theoretically, so the modal components are orthogonal to each other. However, it is impossible to achieve absolute orthogonality between any two IMFs in practical applications; this is because of end effect and mode mixing. Firstly, the end effect of the VMD may result in IMF data diverge at both ends, thus creating a false component. The endpoint effect is more serious in the shorter length signal. In addition, there is no mode mixing in and between the IMFs theoretically, but there still exists mode mixing practically.

\section{Examples of Application}

4.1. Experiment Setup and Signal Acquisition. To evaluate the performance of VMD method, experimental analysis of a rotor system has been carried out in a rotor test rig. As shown in Figure 5, the rotor test rig is composed of electrical motor, shaft, rotor, bearing seat, coupling, sensor support, and foundation. An electrical motor with frequency converter, whose speed is up to $10,000 \mathrm{r} / \mathrm{min}$, is the drive. The 

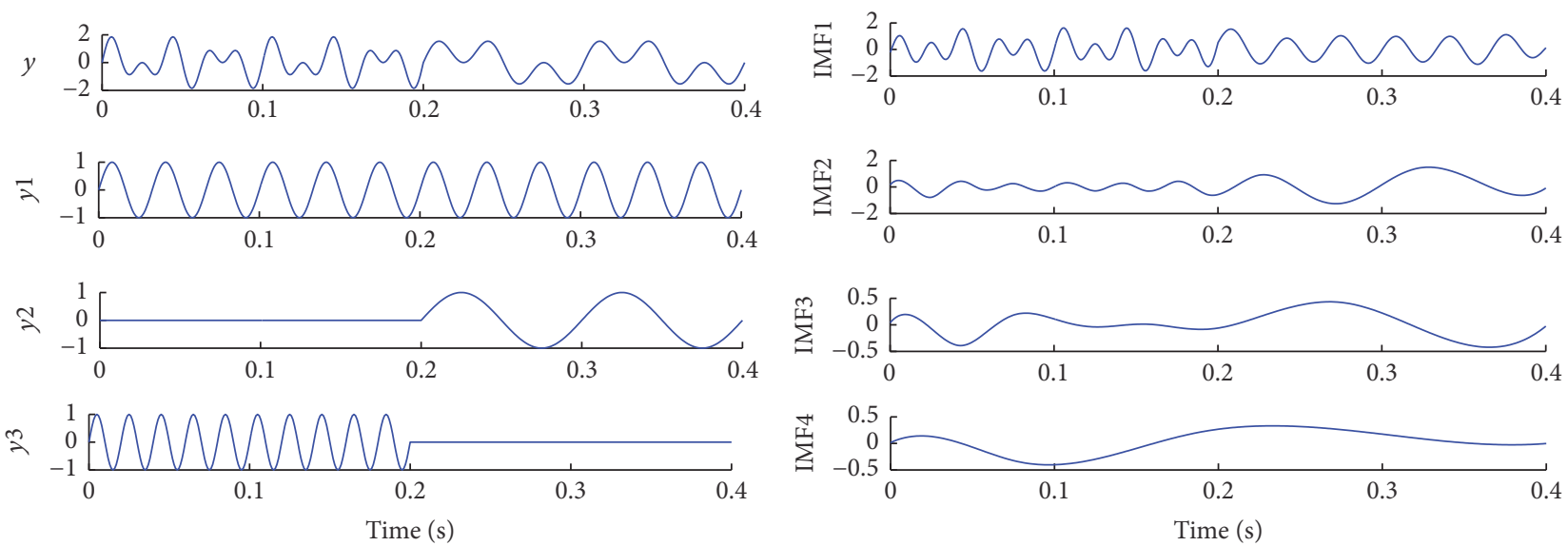

(a) Simulated signal

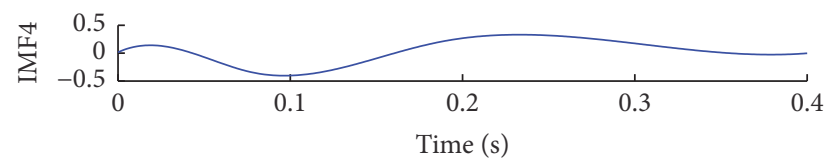

(b) EMD results
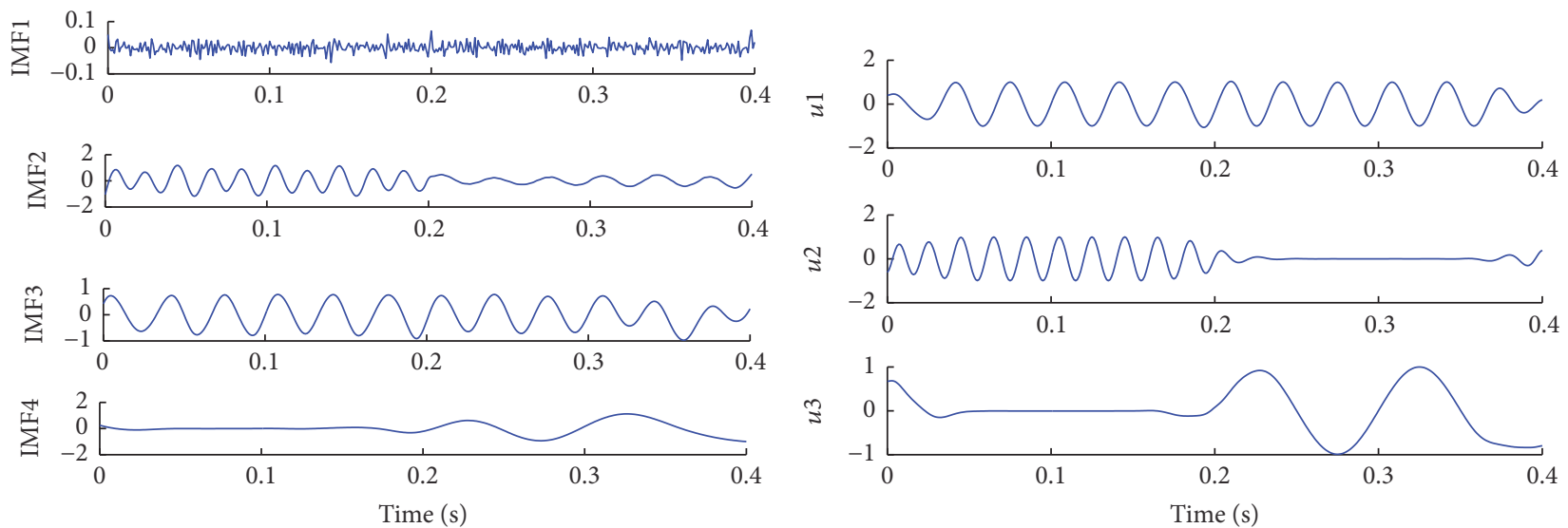

(c) EEMD results

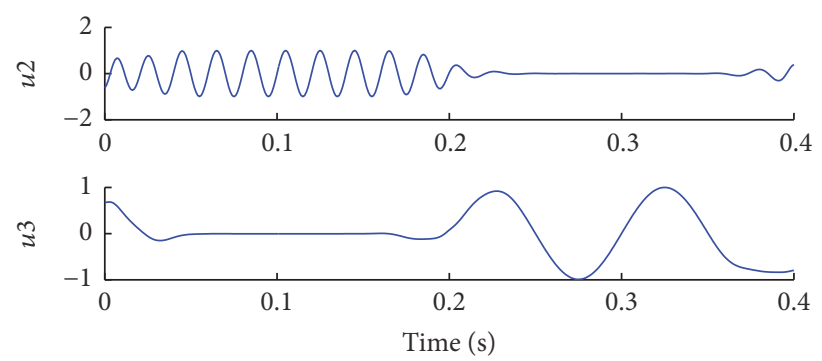

(d) VMD results

FIGURE 2: Comparison results of EMD, EEMD, and VMD.

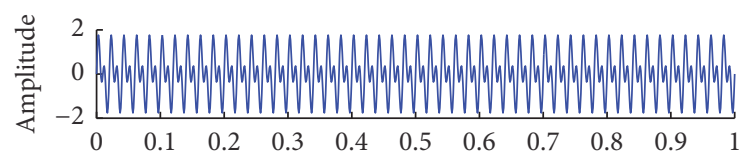

(a) Original signal

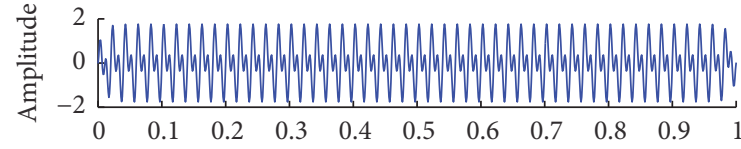

(b) Reconstructed signal

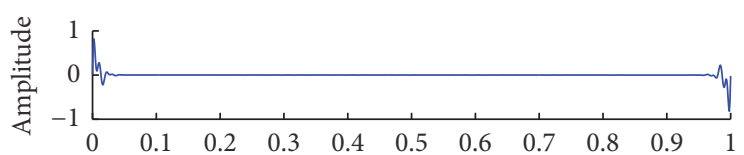

(c) Error

FIGURE 3: Reconfigurability of VMD.

oil film bearing and oil cup were designed to simulate the phenomenon of oil whip.

A phase sensor closed to the motor is fixed to measure the rotating speed. A velocity sensor (ZA-HV-2-5) is fixed on bearing seat in the middle of test rig to measure the vibration of bearing seat. Two eddy current sensors, fixed on sensor support closed to oil film bearing, are used to pick up horizontal and vertical directions displacement of rotor.
4.2. Results and Discussion. The oil film instability of the rotor system usually consists of two stages, oil whirl and oil whip. The whirl is a form of motion in which the rotor rotates around its own axis, while the axis rotates around the center of the bearing. When the rotational speed of the rotor reaches a certain speed, the oil whirl is generated, and the whirling frequency is about half of that speed. When the rotational speed is up to 2 times of the first-order critical speed of rotor system, the oil whirl turns into the oil whip, the amplitude 

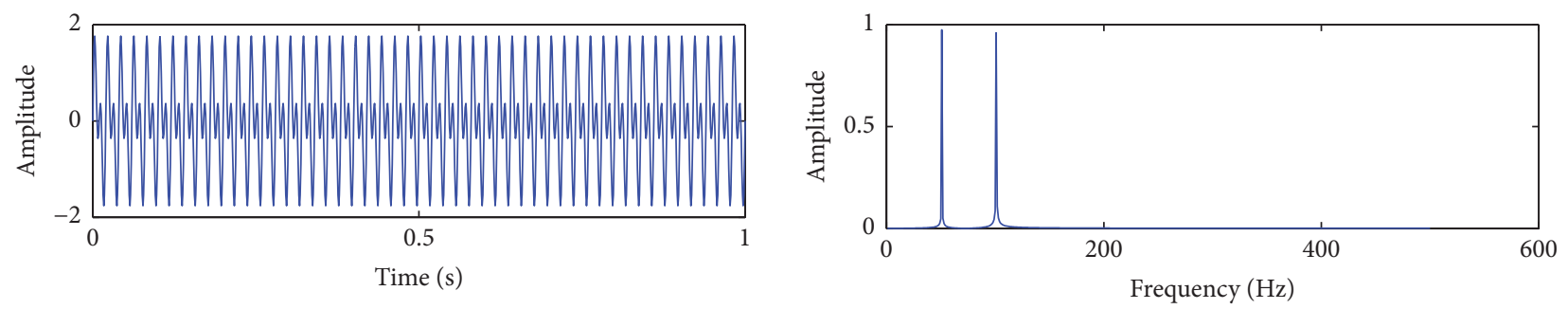

(a) Original signal
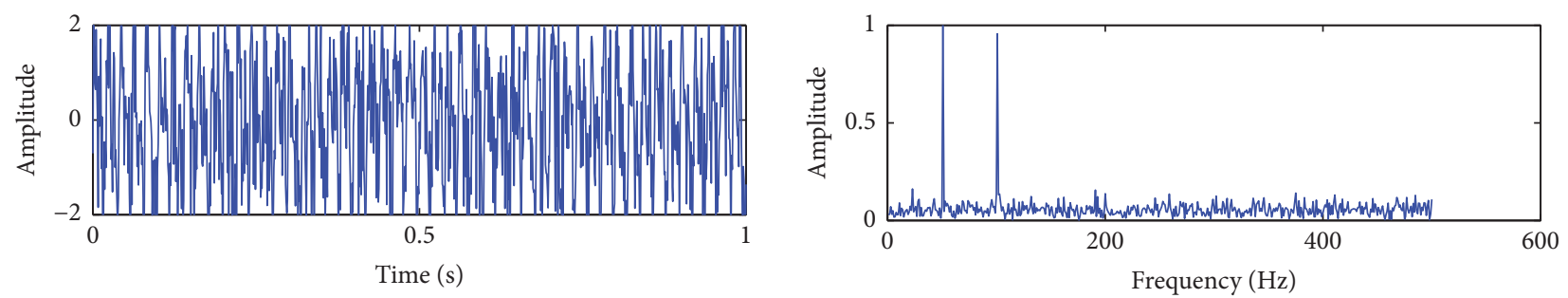

(b) Added noise signal
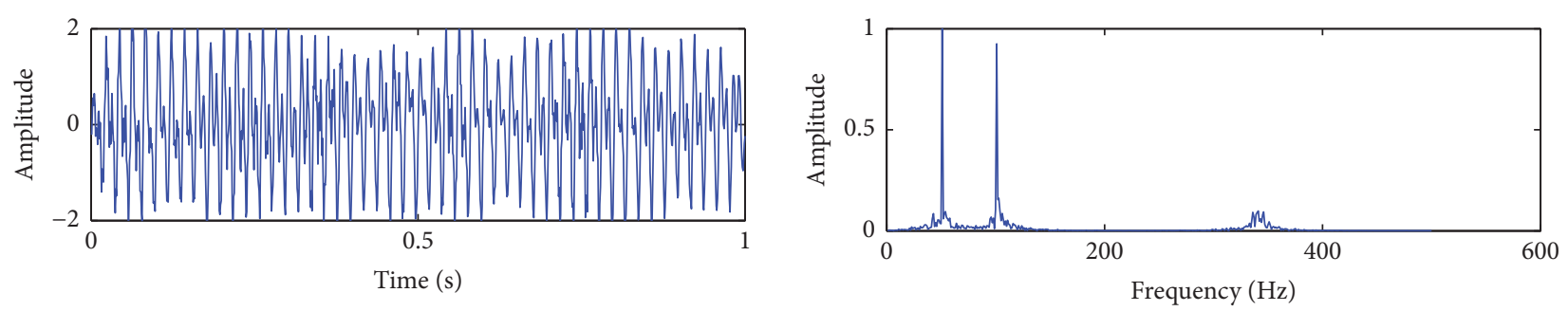

(c) Reconstructed signal

FIgURE 4: The filtering characteristics of VMD.

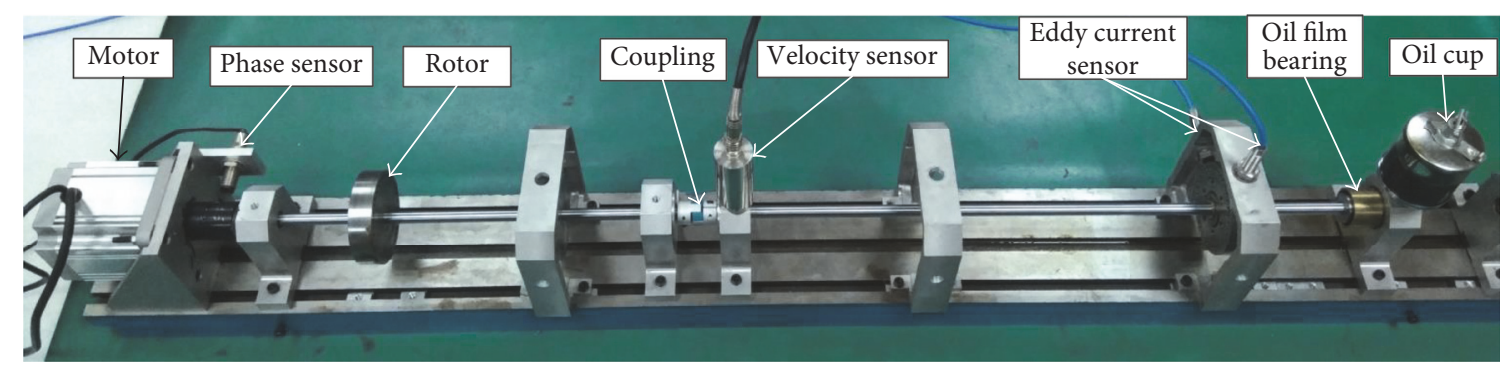

FIGURE 5: Experimental setup and sensor arranged.

of the rotor increases, and the whip frequency stabilizes in a certain value (first-order critical speed of rotor system). The fault feature extraction of oil whirl and oil whip is the main basis to diagnose these type faults.

In the process of oil film instability, the oil whirl occurs near the speed of $3100 \mathrm{r} / \mathrm{min}$. When the rotational speed is up to $4500 \mathrm{r} / \mathrm{min}$, the rotor system test rig shows bigger vibration because of the oil whip.

(1) Oil Whirl. The vibration signal and its spectrum of the oil whirl are shown in Figure 6. In the spectrum, the oil whirl frequency $1 / 2 \mathrm{x}(\approx 26 \mathrm{~Hz})$ and rotating frequency $1 \mathrm{x}(\approx 52 \mathrm{~Hz})$ can be seen. The value of the oil whirl frequency is about half value of rotating frequency and the amplitude is higher than rotating frequency.

Five IMFs decomposed by VMD for oil whirl signal are shown in Figure 7(a), and it is decomposed completely. IMF1 is corresponding to the oil whirl frequency $1 / 2 \mathrm{x}$ and IMF2 is corresponding to rotating frequency $1 \mathrm{x}$. IMF3 is corresponding to $2 \mathrm{x}$. IMF5 is corresponding to $4 \mathrm{x}$. The VMD results not only show the fault feature of oil whirl, but also present more periodicity components.

For comparison, IMFs analyzed by EEMD and EMD are shown in Figures $7(\mathrm{~b})$ and $7(\mathrm{c})$. Although IMF2 and 

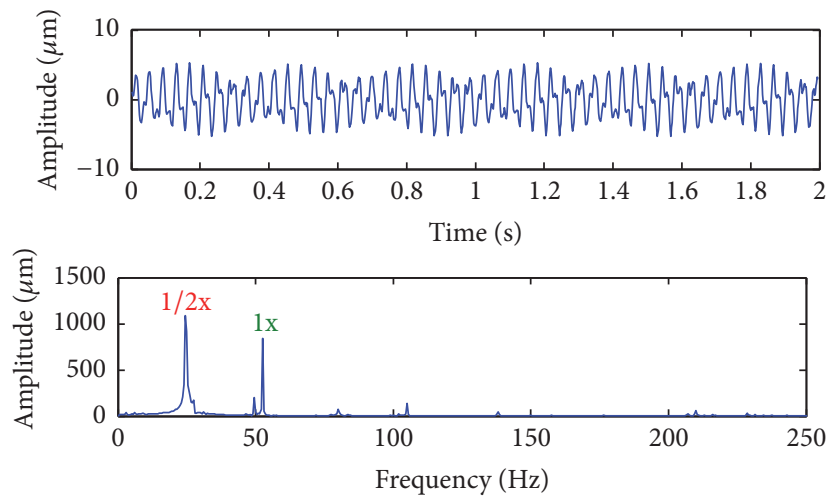

Figure 6: Time Domain waveform and spectrum of oil whirl signal.
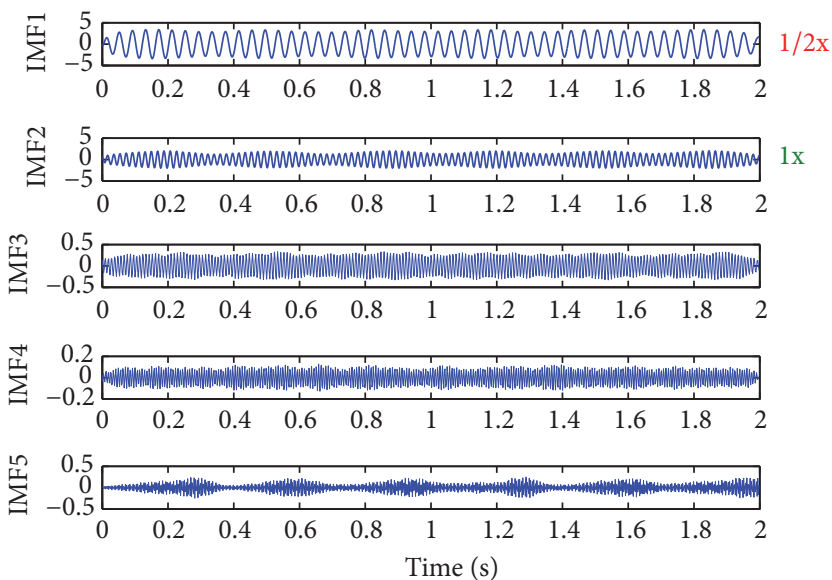

(a) VMD decomposed results

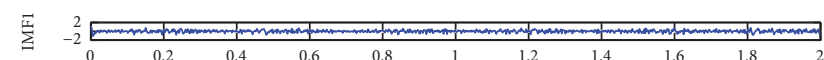

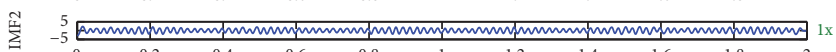

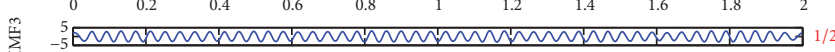

茎 ${ }_{-1}^{1}$

$\sum_{3}^{0.5}{ }_{-0.5}^{0.5}$

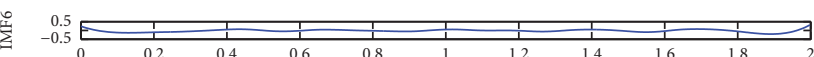

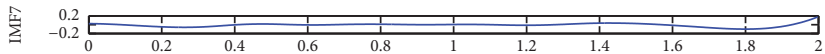

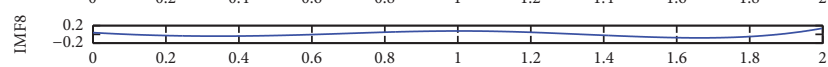

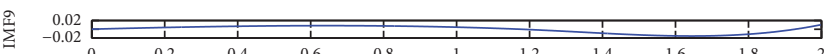

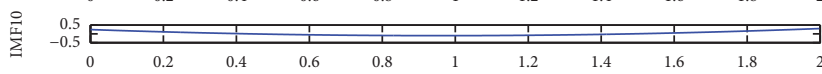

(b) EEMD decomposed results

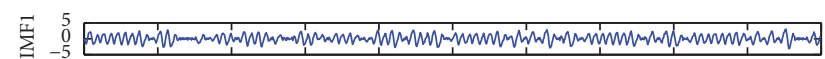

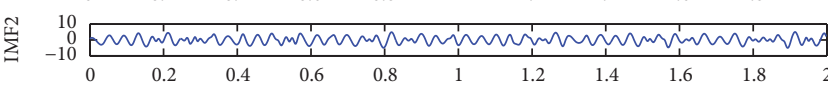
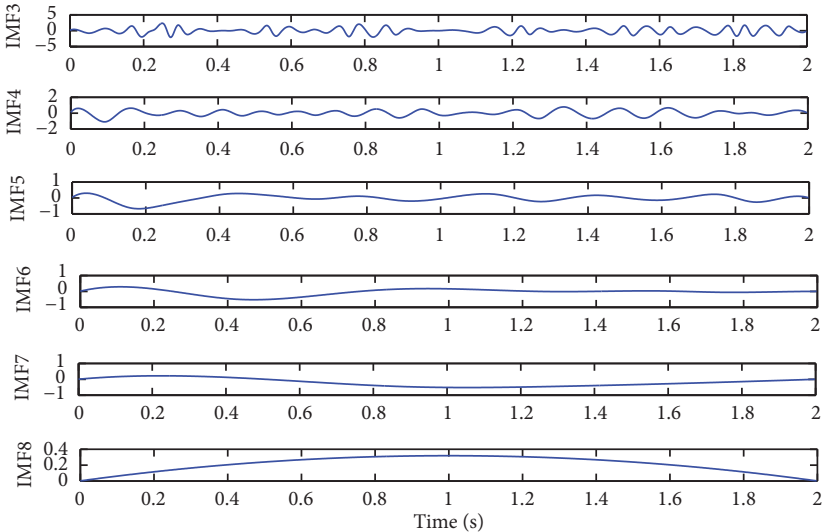

(c) EMD decomposed results

FIGURE 7: VMD, EEMD, and EMD decomposed results of oil whirl signal. 


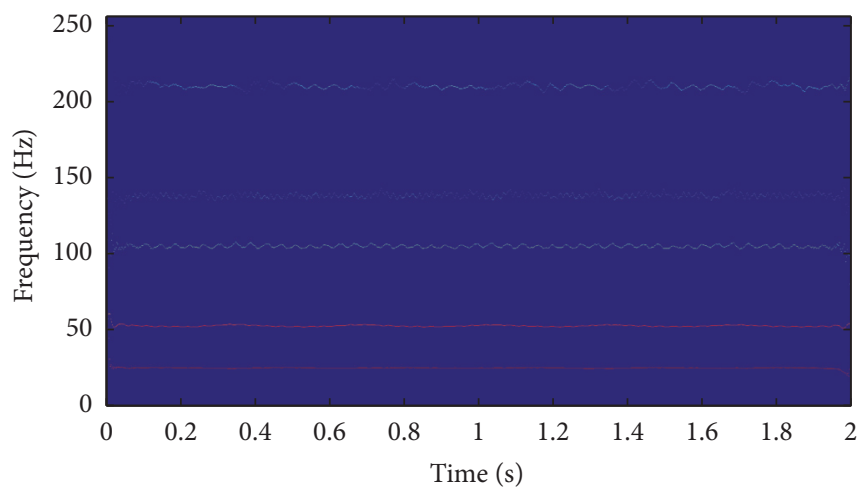

(a) Hilbert spectrum of the VMD results



(b) Hilbert spectrum of the EEMD results

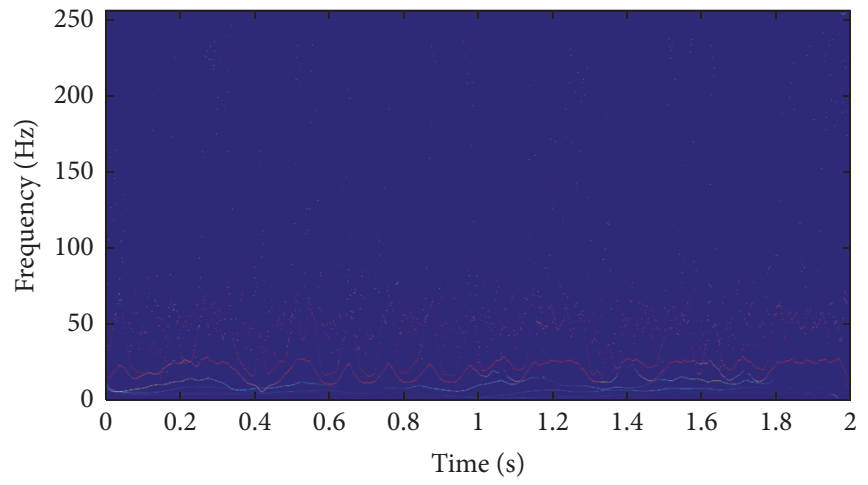

(c) Hilbert spectrum of the EMD results

FIGURE 8: Hilbert spectrum of the VMD, EEMD, and EMD results of oil whirl signal.
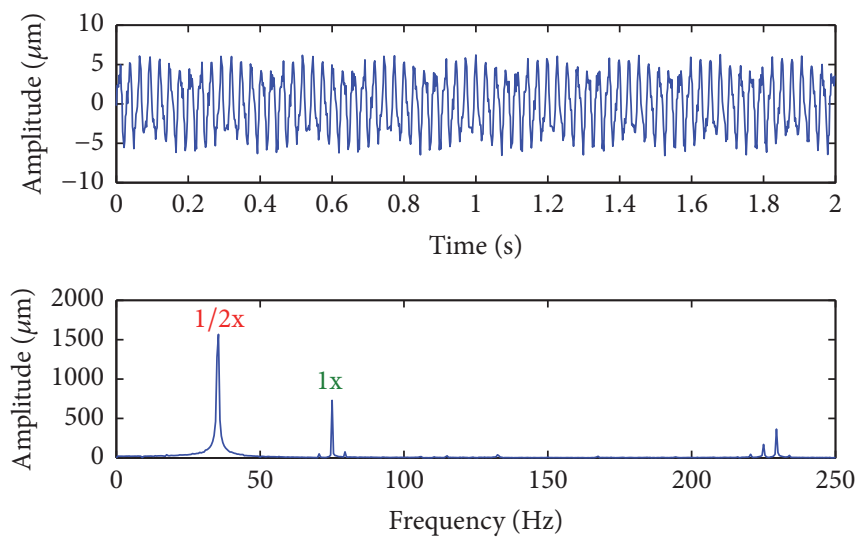

Figure 9: Time domain waveform and spectrum of oil whip signal.

IMF3 decomposed by EEMD are basically the same as the $1 \mathrm{x}$ and $1 / 2 \mathrm{x}$ components in the signal, there still exist mode mixing and additional frequency component in other IMFs. The mixing phenomenon is obvious in EMD results, which cannot show the feature of the oil whirl signal clearly.

Furthermore, the time-frequency spectrum of the VMD, EEMD, and EMD results was analyzed. The Hilbert spectrum of each IMF decomposed by the VMD, EEMD, and EMD is shown in Figure 8 . It can be seen from Figure 8 that the $1 / 2 \mathrm{x}$,
$1 \mathrm{x}, 2 \mathrm{x}, 4 \mathrm{x}$ frequency components of IMFs, decomposed by $\mathrm{VMD}$, are easy to be distinguished. And only $1 / 2 \mathrm{x}$ component can be seen in the EEMD results, while the component of EMD results is very difficult to be resolved.

(2) Oil Whip. The waveform and spectrum of the oil whip signal are shown in Figure 9. In the spectrum, the oil whirl frequency $1 / 2 \mathrm{x}(\approx 37 \mathrm{~Hz})$ and rotating frequency $1 \mathrm{x}(\approx 75 \mathrm{~Hz})$ can be seen. The value of the oil whirl frequency is about half 

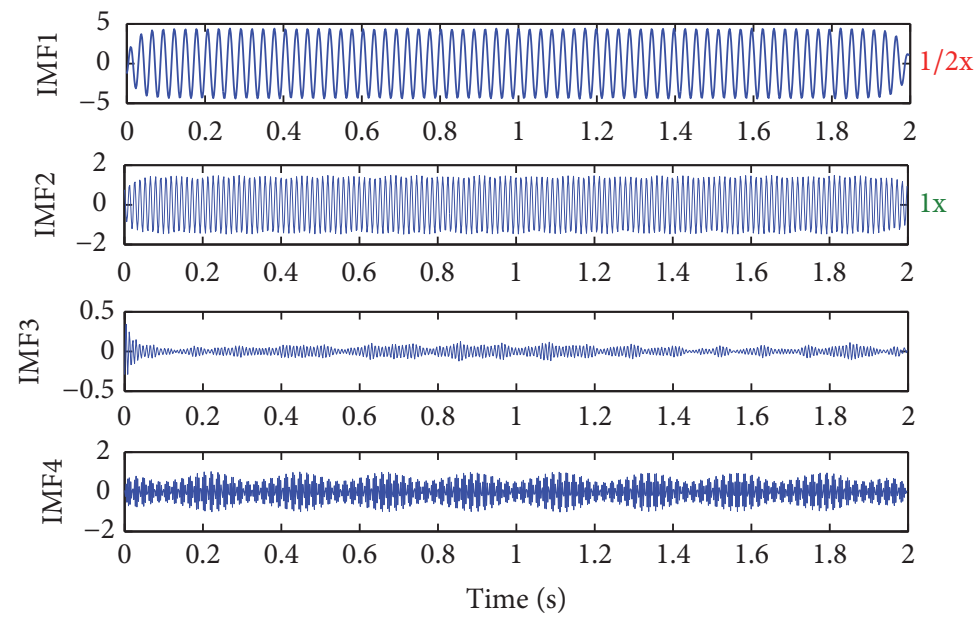

(a) VMD decomposed results

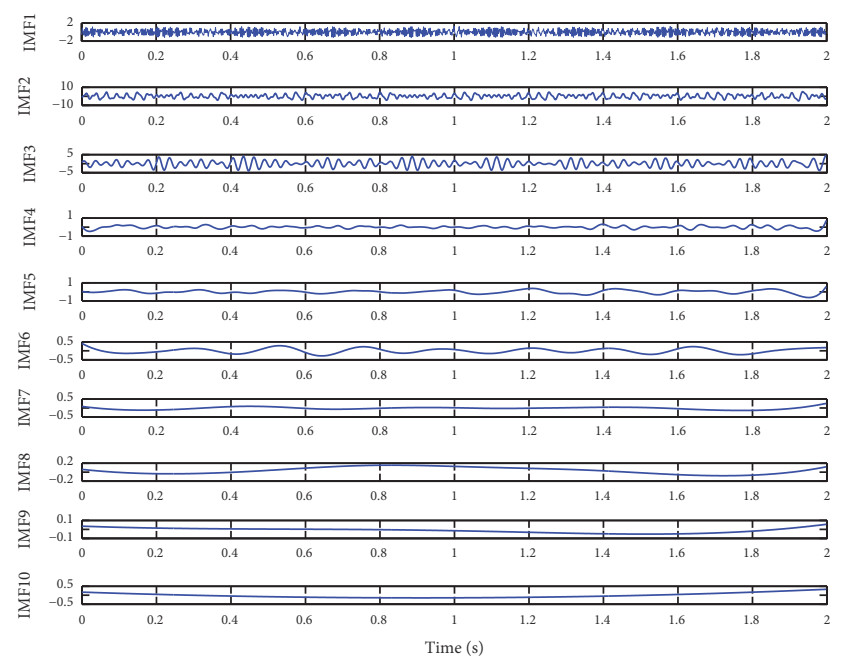

(b) EEMD decomposed results

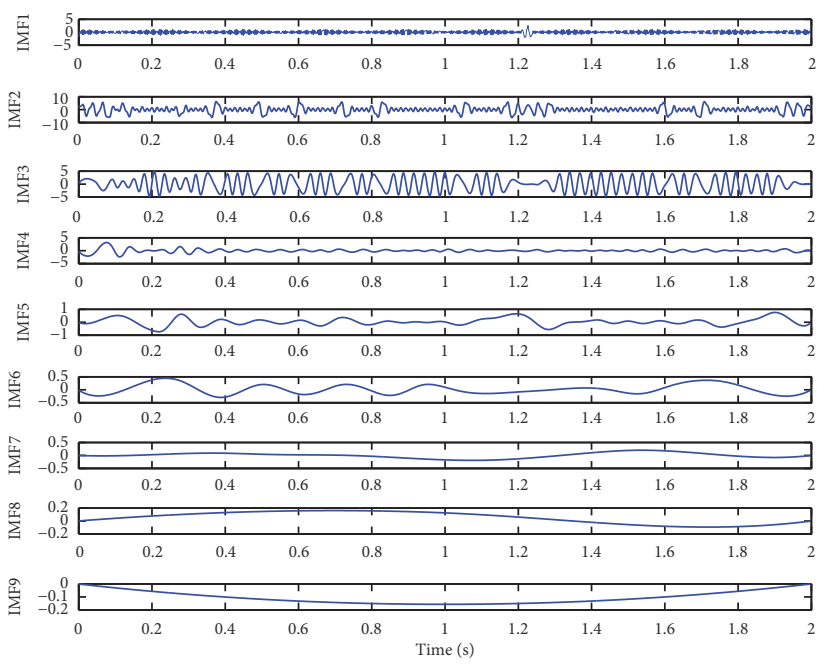

(c) EMD decomposed results

FIGURE 10: VMD, EEMD, and EMD decomposed results of oil whip signal.

value of rotating frequency and the amplitude is higher than rotating frequency.

Four IMFs obtained by VMD for oil whip signal are illustrated in Figure 10(a), and it is decomposed clearly. IMF1 and IMF 2 are corresponding to $1 / 2 \mathrm{x}$ and $1 \mathrm{x}$, respectively, and the $3 \mathrm{x}$ frequency components are also extracted. Figures $10(\mathrm{~b})$ and 10 (c) separately show the IMFs using EEMD and EMD, in which signatures are difficult to be identified.

The Hilbert spectrum of each IMF decomposed by VMD, EEMD, and EMD is shown in Figure 11. As can be seen from Figure 11, all time-frequency representations of each component decomposed by VMD are clearly segregated, while the component of EEMD and EMD results is very difficult to be resolved.

The analysis results in Figures 7, 8, 10, and 11 show better effectiveness of VMD than EEMD and EMD, demonstrating the validity of VMD in detecting rotor faults.

\section{Conclusion}

VMD is a newly developed technique for adaptive signal decomposition, which can decompose a multicomponent signal into a series of quasi-orthogonal intrinsic mode functions simultaneously. Unlike EMD and EEMD, VMD has theoretical support and can solve the problem of mode mixing and end effect more effectively. It also has the characteristic of adaptivity, reconfigurability, smoothing filtering, and orthogonality.

VMD is proposed to determine the fault of rotor systems, and an experimental analysis has been carried out. Comparative analysis results show that VMD method is more effective than EEMD and EMD in fault feature extraction of oil whirl and oil whip, so VMD method has important practical application value for rotor fault diagnosis and should be further considered in the future. 


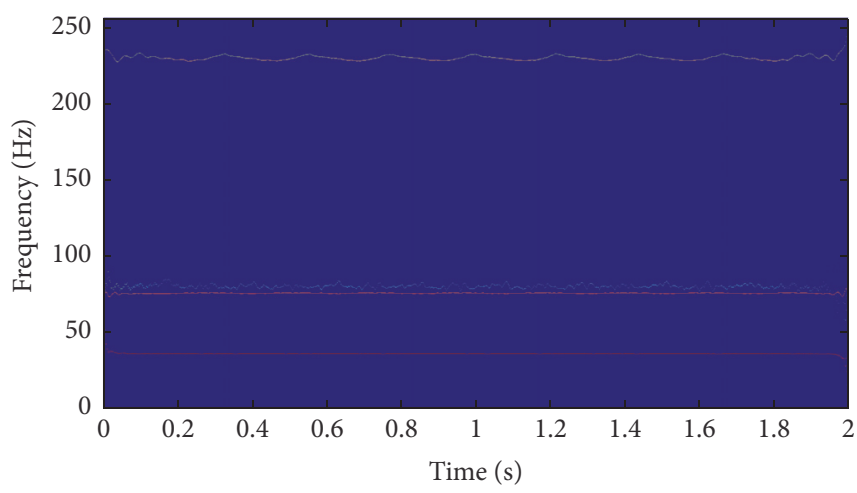

(a) Hilbert spectrum of the VMD results



(b) Hilbert spectrum of the EEMD results

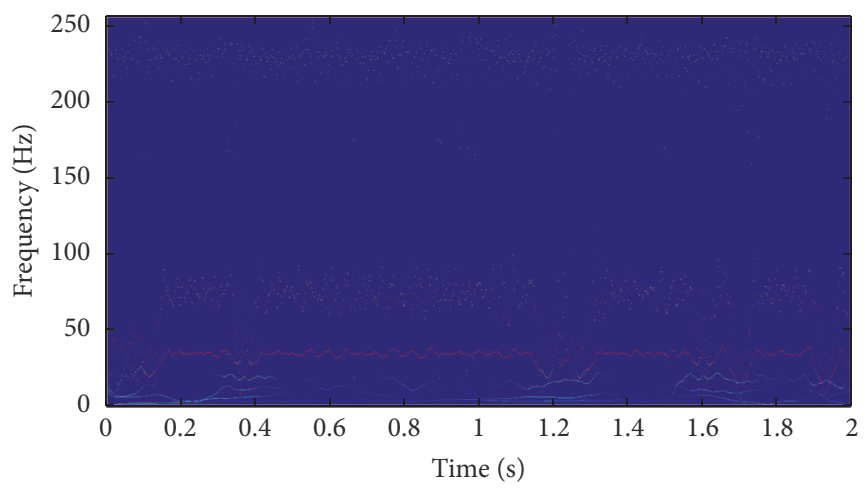

(c) Hilbert spectrum of the EMD results

Figure 11: Hilbert spectrum of the VMD, EEMD, and EMD results of oil whip signal.

\section{Conflicts of Interest}

The authors declare that there are no conflicts of interest regarding the publication of this paper.

\section{Acknowledgments}

This project is sponsored by the grants from the Ministry of Transport Applied Basic Research Project (no. 2013329811360), the National Natural Sciences Foundation of China (no. 51139005, no. 51509194), and the Fundamental Research Funds for the Central Universities (no. 2014-yb018).

\section{References}

[1] D. Yongzuo and Q. Zhiying, "Holo-spectrum analysis of rotating machinery dynamic signals," Journal of Vibration, Measurement \& Diagnosis, vol. 22, no. 2, pp. 81-88, 2002.

[2] Z. Li, Y. Jiang, C. Hu, and Z. Peng, "Recent progress on decoupling diagnosis of hybrid failures in gear transmission systems using vibration sensor signal: a review," Measurement, vol. 90, pp. 4-19, 2016.

[3] D. H. Gonsalves, R. D. Neilson, and A. D. S. Barr, "A study of the response of a discontinuously nonlinear rotor system," Nonlinear Dynamics, vol. 7, no. 4, pp. 451-470, 1995.

[4] N. Bachschmid, P. Pennacchi, and A. Vania, "Identification of multiple faults in rotor systems," Journal of Sound and Vibration, vol. 254, no. 2, pp. 327-366, 2003.
[5] T.-A. Ensslin, M. Frommert, and F.-S. Kitaura, "Information field theory for cosmological perturbation reconstruction and non-linear signal analysis," Physical Review D Particles \& Fields, vol. 80, no. 10, pp. 281-287, 2009.

[6] G. Fele-Žorž, G. Kavšek, Ž. Novak-Antolič, and F. Jager, "A comparison of various linear and non-linear signal processing techniques to separate uterine EMG records of term and preterm delivery groups," Medical and Biological Engineering and Computing, vol. 46, no. 9, pp. 911-922, 2008.

[7] Z. Li, X. Yan, Z. Tian, C. Yuan, Z. Peng, and L. Li, "Blind vibration component separation and nonlinear feature extraction applied to the nonstationary vibration signals for the gearbox multi-fault diagnosis," Measurement: Journal of the International Measurement Confederation, vol. 46, no. 1, pp. 259-271, 2013.

[8] J. Cheng, D. Yu, J. Tang, and Y. Yang, "Local rub-impact fault diagnosis of the rotor systems based on EMD," Mechanism and Machine Theory, vol. 44, no. 4, pp. 784-791, 2009.

[9] N. E. Huang, Z. Shen, S. R. Long et al., "The empirical mode decomposition and the Hilbert spectrum for nonlinear and non-stationary time series analysis," The Royal Society of London. Proceedings. Series A. Mathematical, Physical and Engineering Sciences, vol. 454, no. 1971, pp. 903-995, 1998.

[10] N. E. Huang, M. C. Wu, S. R. Long et al., "A confidence limit for the empirical mode decomposition and Hilbert spectral analysis," Proceedings of the Royal Society A, vol. 459, no. 2037, pp. 2317-2345, 2003.

[11] K. Dragomiretskiy and D. Zosso, "Variational mode decomposition," IEEE Transactions on Signal Processing, vol. 62, no. 3, pp. 531-544, 2014. 
[12] Y. Wang and R. Markert, "Filter bank property of variational mode decomposition and its applications," Signal Processing, vol. 120, pp. 509-521, 2016.

[13] W. Yang, Z. Peng, K. Wei, P. Shi, and W. Tian, "Superiorities of variational mode decomposition over empirical mode decomposition particularly in time-frequency feature extraction and wind turbine condition monitoring," IET Renewable Power Generation, vol. 11, no. 4, pp. 443-452, 2017.

[14] S. Lahmiri, "Comparative study of ECG signal denoising by wavelet thresholding in empirical and variational mode decomposition domains," Healthcare Technology Letters, vol. 1, no. 3, pp. 104-109, 2014.

[15] N. Mohan, S. Sachin Kumar, P. Poornachandran, and K. P. Soman, "Modified variational mode decomposition for power line interference removal in ECG signals," International Journal of Electrical and Computer Engineering, vol. 6, no. 1, pp. 151-159, 2016.

[16] A. Mert, "ECG feature extraction based on the bandwidth properties of variational mode decomposition," Physiological Measurement, vol. 37, no. 4, pp. 530-543, 2016.

[17] P. Shi and W. Yang, "Precise feature extraction from wind turbine condition monitoring signals by using optimised variational mode decomposition," IET Renewable Power Generation, vol. 11, no. 3, pp. 245-252, 2017.

[18] V. Vishnu Pradeep, V. Sowmya, and K. P. Soman, "Variational mode decomposition based multispectral and panchromatic image fusion," International Journal of Control Theory and Applications, vol. 9, no. 16, pp. 8051-8059, 2016.

[19] G. Sun, T. Chen, Z. Wei, Y. Sun, H. Zang, and S. Chen, "A carbon price forecasting model based on variational mode decomposition and spiking neural networks," Energies, vol. 9, no. 1, article 54, 2016.

[20] S. Lahmiri, "Comparing variational and empirical mode decomposition in forecasting day-ahead energy prices," IEEE Systems Journal, no. 99, pp. 1-4, 2015.

[21] A. Upadhyay and R. B. Pachori, "Instantaneous voiced/nonvoiced detection in speech signals based on variational mode decomposition," Journal of the Franklin Institute, vol. 352, no. 7, pp. 2679-2707, 2015.

[22] C. Yi, Y. Lv, and Z. Dang, "A fault diagnosis scheme for rolling bearing based on particle swarm optimization in variational mode decomposition," Shock and Vibration, vol. 2016, Article ID 9372691, 10 pages, 2016.

[23] Z. Lv, B. Tang, Y. Zhou, and C. Zhou, "A novel method for mechanical fault diagnosis based on variational mode decomposition and multikernel support vector machine," Shock and Vibration, vol. 2016, Article ID 3196465, 11 pages, 2016.

[24] Z. Li, Y. Jiang, Q. Guo, C. Hu, and Z. Peng, "Multi-dimensional variational mode decomposition for bearing-crack detection in wind turbines with large driving-speed variations," Renewable Energy, 2016.

[25] N. Huang, H. Chen, G. Cai, L. Fang, and Y. Wang, "Mechanical fault diagnosis of high voltage circuit breakers based on variational mode decomposition and multi-layer classifier," Sensors, vol. 16, no. 11, article 1887, 2016.

[26] Z. H. Wu and N. E. Huang, "Ensemble empirical mode decomposition: a noise-assisted data analysis method," Advances in Adaptive Data Analysis, vol. 1, no. 1, pp. 1-41, 2009. 


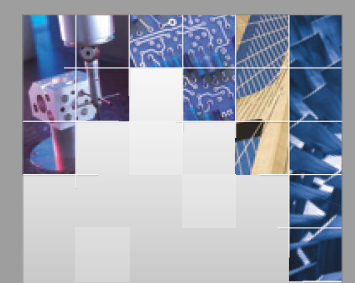

\section{Enfincering}
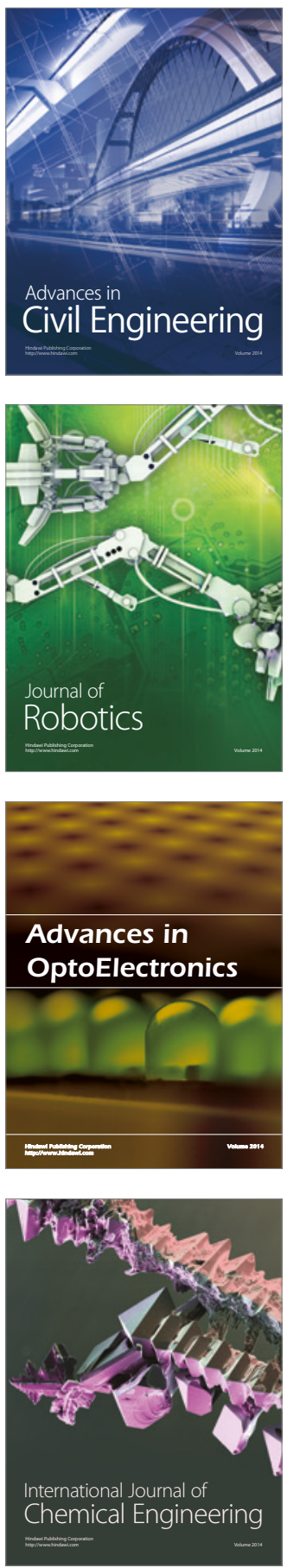

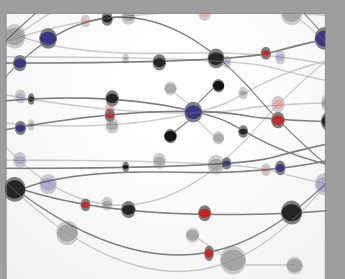

The Scientific World Journal

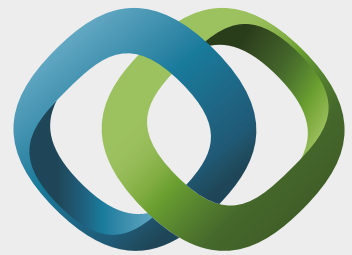

\section{Hindawi}

Submit your manuscripts at

https://www.hindawi.com


\section{Rotating}

Machinery
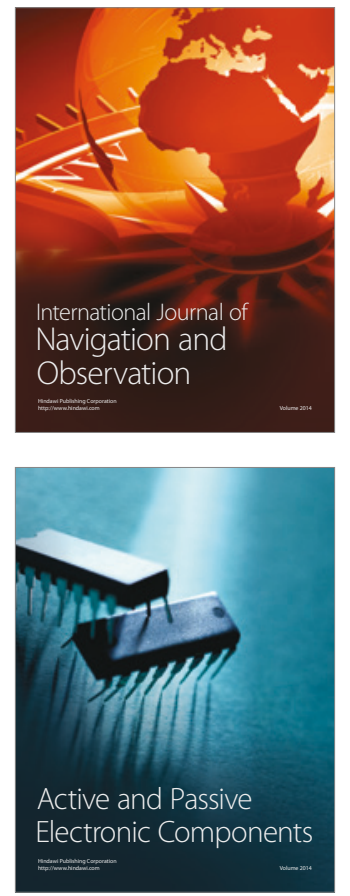
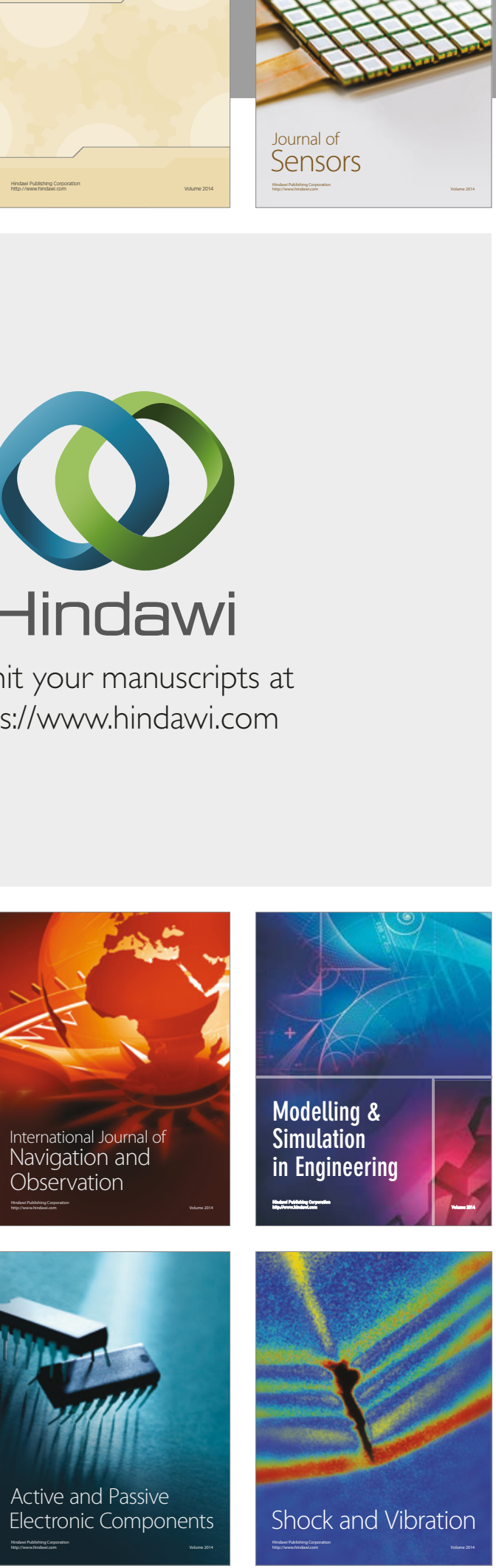
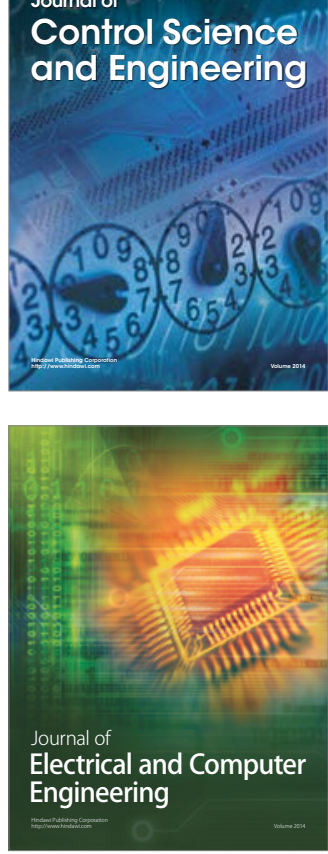

Distributed

Journal of

Control Science

and Engineering
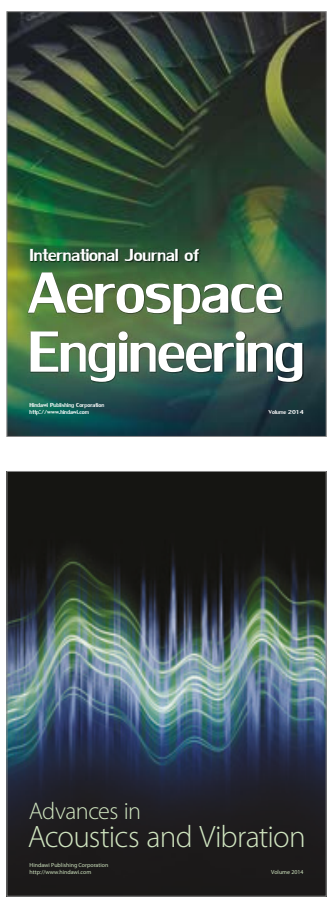

Sensor Networks 